\title{
Plants as Effective Agents in Treating Diabetes and Its Complications
}

\author{
Uma Devi Palanisamy* \\ Department of Biochemistry, Monash University, Malaysia
}

\begin{abstract}
Received: February 20, 2014; Accepted: February 28, 2014; Published: March 03, 2014
*Corresponding author: Uma Devi Palanisamy, Department of Biochemistry, Jeffrey Cheah School of Medicine and Health Sciences, Monash University, Malaysia, Tel: 603 55145840; E-mail: umadevi.palanisamy@monash.edu
\end{abstract}

Diabetes mellitus is a metabolic disease characterized by hyperglycemia resulting from defect in insulin production and or action, or both. It is a global problem that has a significant impact on the health, quality of life, and life expectancy of patients, as well as on the health care system. Diabetes can be classified into type 1 diabetes, Insulin Dependent Diabetes Mellitus (IDDM) and type 2 diabetes, Non-Insulin Dependent Diabetes Mellitus (NIDDM). The vast majority of cases of diabetes fall into type 2 diabetes.

During onset and development of type 2 diabetes, cellular balance of carbohydrate and lipid metabolism is affected by improper glucose metabolism; which leads to elevated postprandial blood glucose levels. One therapeutic approach for treating diabetes is to decrease the post-prandial hyperglycemia by retarding absorption of glucose through inhibition of carbohydrate hydrolyzing enzymes like $\alpha$-glucosidase and $\alpha$-amylase in the digestive tract.

The $\alpha$-glucosidase inhibitors decrease the absorption of carbohydrates from the intestine, resulting in a slower and lower rise in blood glucose throughout the day, especially right after meals. The commercialized alpha glucosidase inhibitors; acarbose, miglitol and voglibose have been used for type 2 diabetes management. The $\alpha$-amylase inhibitors known as 'starch blockers', will attach itself to the carbohydrate molecule thus preventing carbohydrate from breaking down prior to absorption. Thus inhibition of $\alpha$-amylase also reduces the postprandial hyperglycaemia in diabetes.

Long standing hyperglycaemia with diabetes mellitus leads to the formation of advanced glycosylated end products which are involved in the generation of Reactive Oxygen Species (ROS) and causes oxidative damage. Oxidative stress is believed to be a common pathway linking diverse mechanisms for the pathogenesis of complications of diabetes. There is strong evidence for increased levels of indicators of oxidative stress in diabetic individuals suffering from complications. Lipid peroxidation is one of the characteristic features of chronic diabetes which release free radical that induce process leading to oxidative deterioration, which can be prevented by antioxidants. These complications that develop during diabetes state can be retarded by inhibiting a key enzyme involved in the polyol pathway i.e. aldose reductase which in turn can help prevent the formation of advanced glycation endproducts (AGE). The inhibition of aldol reductase (AR) and advanced glycation endproducts (AGE) is yet another mode of diabetes treatment not dependent on the control of blood glucose level, and can be used in the prevention or reduction of certain diabetic complications.

Anti-oxidants are of interest to both food scientists and health professionals. Whilst anti-oxidants are often added to foods to stabilize them and prevent off-flavour development, considerable interest has been expressed for their potential role as therapeutic agents. The powerful antioxidant properties of vitamins $\mathrm{C}, \mathrm{E}$ and b-carotenes are well known. However, there is another group of naturally-occurring inhibitors of oxidation that are receiving a lot of attention lately, namely the phenolics and polyphenolics, which are secondary plant metabolites. Studies have shown that polyphenols are better scavengers of free radicals than vitamins $\mathrm{C}$ and E. Plants which are high in antioxidants play an important role in the treatment of diabetes. Some of the phenolic compounds with high antioxidant properties are known to be involved in the healing process of free radical-mediated diseases including diabetes mellitus. In addition, secondary plant metabolites such as quercetin, have been shown to inhibit aldose reductase and prevent the formation of advanced glycation endproducts (AGE) hence reduce diabetic complicatons.

Much of the interest in plant phenolics are in flavonoids. Flavonoids are some of the most powerful and effective antioxidant compounds available to humans; and since we are unable to produce flavonoids ourselves, we must get them from the food we eat and from supplements. Several studies have shown that normal intake of flavonoids through eating a range of fruits and vegetables is not enough to keep modern levels of free radicals at bay. Food companies are increasingly promoting the health benefits of flavonoids contained in fruits and vegetables. The trend has been to investigate a variety of plants as new potential sources of bioactive flavonoids.

There are more than 200 pure compounds from plants that have been reported to show blood glucose lowering activity. Plants have been suggested as a rich, as yet unexplored source 
of potentially useful anti-diabetic drugs. Recent scientific investigations have confirmed the efficacy of many of these preparations, some of which are remarkably effective. Some such examples include; Pterocarpus, shown to regenerate functional pancreatic beta cells; bitter melon, a hypoglycemic agent composed of mixed steroids that is more potent than the drug tolbutamide, also contains an insulin-like polypeptide, polypeptide-P, which lowers blood sugar levels; gymnema, improves the ability of insulin to lower blood sugar in both
Type 1 and Type 2 diabetes; fenugreek seeds, demonstrated in experimental and clinical studies to have antidiabetic properties and contain the alkaloids trogonelline, nicotinic acid and coumarin.

There are plenty of opportunities in the market to place these extracts because they are natural ingredients with low or no side effects and have a positive reputation due to the use of herbals as traditional products. In addition to their effectiveness, are applicable at a reasonable price. 